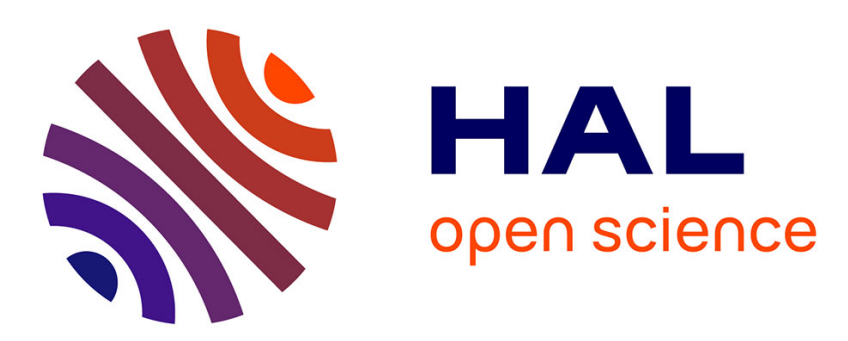

\title{
Metasurface-mediated anisotropic radiative heat transfer between nanoparticles
}

\author{
Yong Zhang, Mauro Antezza, Hing-Liang Yi, He-Ping Tan
}

\section{To cite this version:}

Yong Zhang, Mauro Antezza, Hing-Liang Yi, He-Ping Tan. Metasurface-mediated anisotropic radiative heat transfer between nanoparticles. Physical Review B, 2019, 100, pp.085426. 10.1103/PhysRevB.100.085426 . hal-02267129

\section{HAL Id: hal-02267129 \\ https://hal.science/hal-02267129}

Submitted on 14 Oct 2020

HAL is a multi-disciplinary open access archive for the deposit and dissemination of scientific research documents, whether they are published or not. The documents may come from teaching and research institutions in France or abroad, or from public or private research centers.
L'archive ouverte pluridisciplinaire HAL, est destinée au dépôt et à la diffusion de documents scientifiques de niveau recherche, publiés ou non, émanant des établissements d'enseignement et de recherche français ou étrangers, des laboratoires publics ou privés. 


\title{
Metasurface-mediated anisotropic radiative heat transfer between nanoparticles
}

\author{
Yong Zhang, ${ }^{1,2}$ Mauro Antezza, ${ }^{3,4}$ Hong-Liang Yi $\odot,{ }^{1,2, *}$ and He-Ping Tan ${ }^{1,2}$ \\ ${ }^{1}$ School of Energy Science and Engineering, Harbin Institute of Technology, Harbin 150001, P. R. China \\ ${ }^{2}$ Key Laboratory of Aerospace Thermophysics, Ministry of Industry and Information Technology, Harbin 150001, P. R. China \\ ${ }^{3}$ Laboratoire Charles Coulomb (L2C) UMR 5221 CNRS-Université de Montpellier, F- 34095 Montpellier, France \\ ${ }^{4}$ Institut Universitaire de France, 1 rue Descartes, F-75231 Paris, France
}

(Received 20 March 2019; revised manuscript received 12 June 2019; published 16 August 2019)

\begin{abstract}
Metasurfaces, the two-dimensional (2D) counterpart of metamaterials, have recently attracted a great deal of attention due to their amazing properties, including negative refraction, hyperbolic dispersion, and manipulation of the evanescent spectrum. In this work, a theory model is proposed for the near field radiative heat transfer (NFRHT) between two nanoparticles in the presence of an anisotropic metasurface. Specifically, the metasurface is modeled as an array of graphene strips (GS), which is an ideal platform to implement any metasurface topology, ranging from isotropic to hyperbolic propagation. The NFRHT between two nanoparticles are significantly amplified when they are placed in the proximity of the GS, and regulated over several orders of magnitude. In this configuration, the anisotropic surface plasmon polaritons (SPPs) supported by the GS are excited and provide a new channel for the near-field energy transport. The dependence of conductance between two nanoparticles on the orientation, the structure parameters, the chemical potential of the GS, and the interparticle or the particle-surface distances are analyzed by clearly identifying the characteristics of the anisotropic SPPs such as dispersion relations, propagation length, and decay length. These results demonstrate a powerful method to regulate the energy transport in particle systems, and create a way to explore the anisotropic optical properties of the metasurface based on the measured heat transfer properties.
\end{abstract}

DOI: 10.1103/PhysRevB.100.085426

\section{INTRODUCTION}

When two objects are brought in proximity to each other, the radiative heat transfer (RHT) between them may be significantly enhanced in the near field. This near field enhancement is caused by the tunneling effect of evanescent modes, especially when surface modes such as surface plasmon polaritons (SPPs) or surface phonon polaritons (SPhPs), are excited [1-9]. The huge radiative heat flux in the near field allows various applications such as thermophotovoltaics [10], thermal rectification [11], and information processing [12]. Since a large number of heat fluxes are of critical importance in these appealing applications, the ability to control such near-field radiative heat transfer (NFRHT) has attracted much attention in nanoscale science during the past years [13-19].

Typically, a remarkable theoretical effort in this domain has been devoted to the study of RHT between two or more particles [20-27]. One of the most popular simplifications is the dipole approximation where NFRHT is computed for point-like particles. This assumption considerably simplifies the calculations. Previous work has been focused on the active control of the cooling and heating of nanoparticles, either in vacuum or in the proximity of an interface, as well as in the temperature profile within a collection of nanoparticles. Most recently, Dong et al. [28] and Messina et al. [29] addressed the role of surface waves in the energy transport through two or more nanoparticles placed in the proximity

*Corresponding author: yihongliang@hit.edu.cn of a planar interface. It has been shown that the presence of a planar substrate supporting a surface resonance enhances the NFRHT by orders of magnitude at long distances. In addition, Asheichyk et al. [30] studied the RHT between two nanoparticles placed inside a two-plate cavity. It is found that the presence of plates is not additive in the sense that the results for two plates are distinct from the ones for a single plate studied in Refs. [28,29].

Until now, the planar substrates considered are those supporting isotropic surface plasmon SPPs or SPhPs). In this work, we focus on the NFRHT between nanoparticles in the presence of an anisotropic metasurfaces. Metasurfaces, the two-dimensional (2D) counterpart of metamaterials, have recently attracted a great deal of attention due to their amazing properties such as negative refraction, hyperbolic dispersion, manipulation of the evanescent spectrum, drastic emission enhancement, cloaking, and electromagnetic transparency [31-39]. Unlike three-dimensional (3D) metamaterials, 2D metasurfaces would enable more ambitious applications with increased resolution and simpler excitation, processing, and retrieval of light via near-field techniques [40,41]. The RHT between planar natural anisotropic materials or patterned structures have been recently studied [42-44]. In this work, we include the contribution of the metasurface by imposing the boundary conditions described by the reflected dyadic Green's function. Specifically, we consider graphene strips (GS) in this work. The homogenization of such a metasurface in the subwavelength approximation (where the period is far less than the wavelength, $L \ll \lambda$ ) can be performed using the effective medium theory based on the electrostatic 


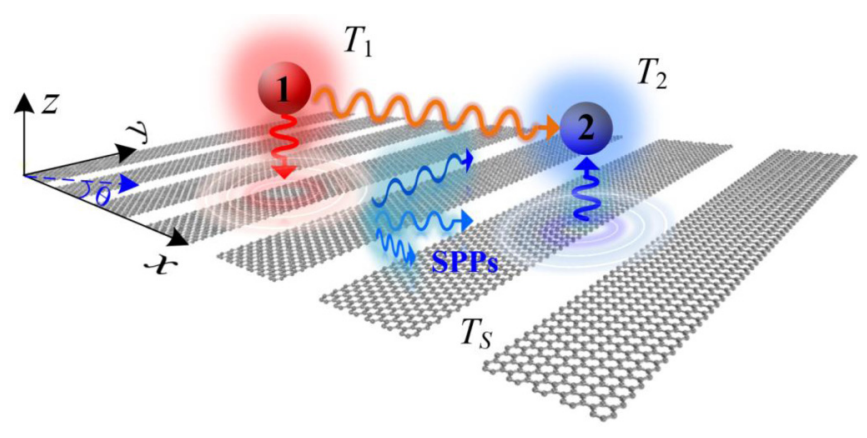

FIG. 1. Radiative heat transfer between two nanoparticles above an array of graphene strips.

approach [45]. It is shown that the GS can propagate plasmons along large distances compared to the plasmons' wavelength [46].

We observe that the anisotropic SPPs supported by the GS have a large effect on NFRHT and can increase these quantities by several orders of magnitude compared to isolated objects. The physics behind this effect is studied both in terms of the distributions of the Green's function with respect to the wave vector and the equal-frequency contours of the light dispersion in the metasurface, in order to identify the role played by the anisotropic surface mode. Moreover, we show that the NFRHT in our configuration exhibits great tunable features by varying the structures or optical parameters of the GS, and it is related to the modification of the anisotropic SPPs excited on the GS.

The paper is structured as follows. In Sec. II, we introduce the geometry of our system, define the Green's function in the presence of an anisotropic metasurface, and give the expression of the heat flux between the two nanoparticles. Section III introduces the optical properties of the GS, and studies the RHT between two nanoparticles placed in the proximity of the metasurface. We show that the presence of GS significantly modifies the RHT between two nanoparticles. To understand the physical origin of the results we discuss the Green's function in the wave-vector space and the energy density in the spatial space. Sections IV and V are dedicated to the effects of the interdistance, particle-GS distance, and the chemical potential. The propagation and decay lengths of the anisotropic SPPs are adopted to interpret the results. Finally, in Sec. VI, we give some concluding remarks and perspectives.

\section{THEORETICAL ASPECTS}

To start, let us consider the RHT between two nanoparticles in the presence of a metasurface as shown in Fig. 1. Note that the metasurface is an array of graphene strips. However, the theory derived below can be applied to any kind of 2D metasurface. We suppose the nanoparticles are isotropic, linear, nonmagnetic, and the sizes of the nanoparticles are much smaller than the thermal wavelength $\lambda_{T}=c \hbar /\left(k_{B} T\right)$ so that all individual objects can be modeled as simple radiating electrical dipoles.

We note that by using external reservoirs, particles 1 and 2, as well as the metasurface, could be kept at fixed temperatures $T_{1}, T_{2}$, and $T_{S}$, respectively, in time. We assume now that the whole system is thermalized at a given temperature $T$, but the temperature of particle 1 is slightly increased to $T_{1}=$ $T+\Delta T$. Now, since particle 2 has no net heat exchange with the metasurface (both are at the same temperature $T_{2}=$ $T_{S}=T$ ), the only net heat exchanged by particle 2 is with particle 1. As the temperature difference $\Delta T$ tends to 0 , we get the radiative heat transfer conductance $h$ between these two particles. This is the quantity we are going to discuss in the following, addressing in particular the question of how $h$ is mediated by the presence of the anisotropic metasurface. We note that particle 1 will also exchange a portion of its heat with the metasurface exclusively, and the value of this heat transfer will not affect the energy transferred from particle 1 to particle 2 . Thus, in this work, the metasurface purely acts as a boundary condition, modifying the way in which the direct exchange between the particles in vacuum takes place. The conductance $h$ between two identical nanoparticles at a temperature of $T$ can be conveniently expressed in terms of the Green's function describing the system as [21,29]

$$
h=4 \int_{0}^{+\infty} \frac{d \omega}{2 \pi} \hbar \omega k_{0}^{4} n^{\prime}(\omega, T) \chi^{2} \operatorname{Tr}\left(\mathcal{G G}^{*}\right),
$$

where $k_{0}=\omega / c$ is the wave vector in vacuum and $\chi(\omega)=$ $\operatorname{Im}[\alpha(\omega)]-k_{0}^{3}|\alpha(\omega)|^{2} / 6 \pi$ denotes the modified electric frequency-dependent polarizability. In the limit $R \ll \delta$ (with $\delta$ being the skin depth of the given material), $\alpha$ can be written in the well-known Clausius-Mossoti form [47]

$$
\alpha(\omega)=4 \pi R^{3} \frac{\varepsilon(\omega)-1}{\varepsilon(\omega)+2},
$$

with $R$ and $\varepsilon(\omega)$ being the radius and the electric permittivity of the particle, respectively. In this work, we will assume that the two nanoparticles are identical spheres of radius $R=$ $5 \mathrm{~nm}$.

$\mathcal{G}$ in Eq. (1) denotes the dyadic Green's tensor of the full system, which is written in terms of Green's tensor $\mathbb{G}$ as

$$
\mathcal{G}=\mathbb{M}^{-1} \mathbb{G}
$$

where $\mathbb{M}=\mathbf{I}-k_{0}^{4} \alpha_{1} \alpha_{2} \mathbb{G} \mathbb{G}^{T}$ represents the multiple reflections between the two nanoparticles. $n^{\prime}(\omega, T)$ denotes the derivative with respect to $T$ of the Bose-Einstein distribution $n(\omega, T)=\left(e^{\hbar \omega / k_{B} T}-1\right)^{-1}$.

As the two nanoparticles are placed on the same side of the metasurface, the Green's tensor can be written as [48]

$$
\mathbb{G}=\mathbb{G}_{0}+\mathbb{G}_{R}
$$

i.e., separated into a vacuum contribution and a reflected part, the latter of which depends on the metasurface reflection matrix and goes to zero in the absence of the metasurface. The vacuum contribution to the Green's function is

$$
\mathbb{G}_{0}=\frac{e^{i k_{0} d}}{4 \pi k_{0}^{2} d^{3}}\left(\begin{array}{lll}
a & 0 & 0 \\
0 & b & 0 \\
0 & 0 & b
\end{array}\right)
$$

where $d$ is the distance between the two nanoparticles, $a=2-2 i k_{0} d$ and $b=k_{0}^{2} d^{2}+i k_{0} d-1$. 
The reflected electric-electric Green's function $\mathbb{G}_{R, E E}$ for the 2D anisotropic surface is expressed as [49,50]

$$
\mathbb{G}_{R, E E}\left(\mathbf{r}_{i}, \mathbf{r}_{j}, \omega\right)=\frac{i}{8 \pi^{2}} \int_{-\infty}^{\infty} d k_{x} \int_{-\infty}^{\infty}\left(r_{s s} \mathbf{M}_{s s}+r_{p s} \mathbf{M}_{p s}+r_{s p} \mathbf{M}_{s p}+r_{p p} \mathbf{M}_{p p}\right) e^{i\left[k_{x}\left(x_{i}-x_{j}\right)+k_{y}\left(y_{i}-y_{j}\right)\right]} e^{i k_{z}\left|z_{i}+z_{j}\right|} d k_{y},
$$

where $\mathbf{r}_{i}=x_{i} \vec{e}_{x}+y_{i} \vec{e}_{y}+z_{i} \vec{e}_{z}$ denotes the position of particle

$$
\begin{aligned}
& \mathbf{M}_{s s}=\frac{1}{k_{z} k_{\rho}^{2}}\left(\begin{array}{ccc}
k_{y}^{2} & -k_{x} k_{y} & 0 \\
-k_{x} k_{y} & k_{x}^{2} & 0 \\
0 & 0 & 0
\end{array}\right), \quad \mathbf{M}_{p p}=\frac{k_{z}}{k_{0}^{2} k_{\rho}^{2}}\left(\begin{array}{ccc}
-k_{x}^{2} & -k_{x} k_{y} & -k_{x} k_{\rho}^{2} / k_{z} \\
-k_{x} k_{y} & -k_{y}^{2} & -k_{y} k_{\rho}^{2} / k_{z} \\
k_{x} k_{\rho}^{2} / k_{z} & k_{y} k_{\rho}^{2} / k_{z} & k_{\rho}^{4} / k_{z}^{2}
\end{array}\right), \\
& \mathbf{M}_{s p}=\frac{1}{k_{0} k_{\rho}^{2}}\left(\begin{array}{ccc}
-k_{x} k_{y} & -k_{y}^{2} & -k_{y} k_{\rho}^{2} / k_{z} \\
k_{x}^{2} & k_{x} k_{y} & k_{x} k_{\rho}^{2} / k_{z} \\
0 & 0 & 0
\end{array}\right), \quad \mathbf{M}_{p s}=\frac{1}{k_{0} k_{\rho}^{2}}\left(\begin{array}{ccc}
k_{x} k_{y} & -k_{x}^{2} & 0 \\
k_{y}^{2} & -k_{x} k_{y} & 0 \\
-k_{y} k_{\rho}^{2} / k_{z} & k_{x} k_{\rho}^{2} / k_{z} & 0
\end{array}\right),
\end{aligned}
$$

where $k_{0}=\sqrt{k_{x}^{2}+k_{y}^{2}+k_{z}^{2}}$ and $k_{\rho}=\sqrt{k_{x}^{2}+k_{y}^{2}}$ are the vacuum and lateral wave vectors, respectively. The tensor reflection coefficient $\mathbf{R}$ related to incident ' $s$ ' and ' $p$ ' polarized waves is $[45,49]$

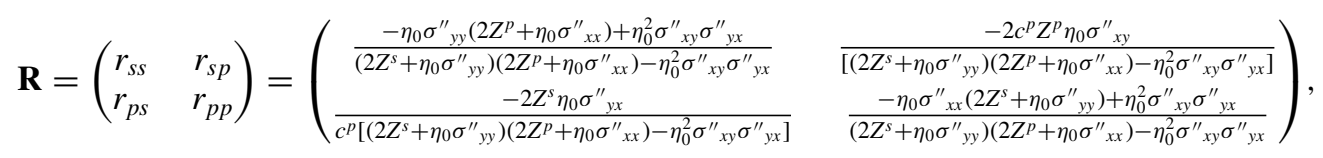

where $\eta_{0}$ is the free-space impedance. $Z^{s}=k_{z} / k_{0}, Z^{p}=k_{0} / k_{z}$ and $c^{p}=k_{z} / k_{0}$. Furthermore, $\sigma^{\prime \prime}$ denotes the conductivity tensor in the wave-vector space $[45,51]$,

$$
\sigma^{\prime \prime}=\left(\begin{array}{ll}
\sigma^{\prime \prime}{ }_{x x} & \sigma^{\prime \prime}{ }_{x y} \\
\sigma^{\prime \prime}{ }_{y x} & \sigma^{\prime \prime}{ }_{y y}
\end{array}\right)=\frac{1}{k_{\rho}^{2}}\left(\begin{array}{ll}
k_{x}^{2} \sigma_{x x}^{\prime}+k_{y}^{2} \sigma_{y y}^{\prime}+k_{x} k_{y}\left(\sigma_{x y}^{\prime}+\sigma_{y x}^{\prime}\right) & k_{x}^{2} \sigma_{x y}^{\prime}-k_{y}^{2} \sigma_{y x}^{\prime}+k_{x} k_{y}\left(\sigma_{y y}^{\prime}-\sigma_{x x}^{\prime}\right) \\
k_{x}^{2} \sigma_{y x}^{\prime}-k_{y}^{2} \sigma_{x y}^{\prime}+k_{x} k_{y}\left(\sigma_{y y}^{\prime}-\sigma_{x x}^{\prime}\right) & k_{x}^{2} \sigma_{y y}^{\prime}+k_{y}^{2} \sigma_{x x}^{\prime}-k_{x} k_{y}\left(\sigma_{x y}^{\prime}+\sigma_{y x}^{\prime}\right)
\end{array}\right),
$$

where $\sigma^{\prime}$ is the conductivity tensor of the metasurface due to the counterclockwise rotation angle $\theta$ with respect to the spatial coordinates.

$$
\boldsymbol{\sigma}^{\prime}=\mathbf{R}^{\mathrm{T}}\left[\begin{array}{cc}
\sigma_{x x} & 0 \\
0 & \sigma_{y y}
\end{array}\right] \mathbf{R}
$$

where $\mathbf{R}=\left[\begin{array}{cc}\cos \theta & -\sin \theta \\ \sin \theta & \cos \theta\end{array}\right]$.

\section{ANISOTROPIC SPPS MEDIATED HEAT TRANSFER BETWEEN TWO NANOPARTICLES}

We now dispose of all the tools needed to calculate the conductance in our system. To understand the mechanism we want to address, the metasurface in this work is modeled as an array of densely packed GS [see Fig. 2(a)] for two reasons: first, it is an ideal platform to implement any metasurface topology, ranging from isotropic to hyperbolic propagation, and going through the extremely anisotropic $\sigma$-near zero case [41]; second, the chemical potential of graphene and the structure parameters, can affect the optical properties of the surface, offering potential routes toward passive or active control of NFRHT. As the strip periodicity $W$ is far less than the plasmons' wavelength $\lambda_{S P P s}$, i.e., $W \ll \lambda_{S P P S}$, the in-plane effective conductivity tensor $\sigma^{\text {eff }}$ of an array of densely packed GS can be analytically derived using the effective medium approach (EMA) [41] as

$$
\sigma_{x x}^{\text {eff }}=\frac{W \sigma \sigma_{C}}{\sigma_{C} P+\sigma(W-P)}, \quad \sigma_{y y}^{\text {eff }}=\sigma \frac{P}{W},
$$

where $P$ is the ribbon width, $\sigma$ is the graphene conductivity given by [52] and $\sigma_{C}=-i \omega \varepsilon_{0} W /(\pi \ln \{\csc [0.5 \pi(1-f)]\})$ is an effective conductivity related to the near-field coupling between adjacent strips obtained through an electrostatic approach, in which $f$ is the filling ratio defined as $P / W$.

As anticipated, we will mainly compare the conductance in vacuum or in the presence of a graphene sheet to that in the presence of the GS. We choose nanoparticles made of silicon carbide ( $\mathrm{SiC})$, a typical polar dielectric material, the dielectric function of which can be described by the Drude-Lorentz model [53]

$$
\varepsilon(\omega)=\varepsilon_{\infty} \frac{\omega_{L}^{2}-\omega^{2}-i \Gamma \omega}{\omega_{T}^{2}-\omega^{2}-i \Gamma \omega},
$$

with high-frequency dielectric constant $\varepsilon_{\infty}=6.7$, longitudinal optical frequency $\omega_{L}=1.83 \times 10^{14} \mathrm{rad} / \mathrm{s}$, transverse optical frequency $\omega_{T}=1.49 \times 10^{14} \mathrm{rad} / \mathrm{s}$, and damping $\Gamma=$ $8.97 \times 10^{11} \mathrm{rad} / \mathrm{s}$. It is stressed that the expression of the electric polarizability given in Eq. (2) predicts nanoparticle resonance frequency $\omega_{r}$ corresponding asymptotically to the condition $\varepsilon(\omega)+2=0$, which for SiC gives $\omega_{r}=1.756 \times$ $10^{14} \mathrm{rad} / \mathrm{s}$. It is well known that for dielectric nanoparticles, the electric contribution dominates the heat transfer. Thus for SiC nanoparticles, we only consider the electric contribution.

In this section, we consider NFRHT between two nanoparticles. As shown in Figs. 1 and 2(a), we put the GS and nanoparticles on the plane of $x-y$ and the $x$ axis, respectively. Due to the anisotropic structure of the GS, we examine the rotation angle $\theta$ of the GS on the RHT between nanoparticles, which is defined as the counterclockwise rotation angle with respect to the $x$ axis. Initially, we set the GS parallel to the $y$ axis for $\theta=0^{\circ}$. The interparticle and particle-GS distances are denoted by $d$ and $z$, respectively. More importantly, it should also be noted that the accuracy of the EMA might 


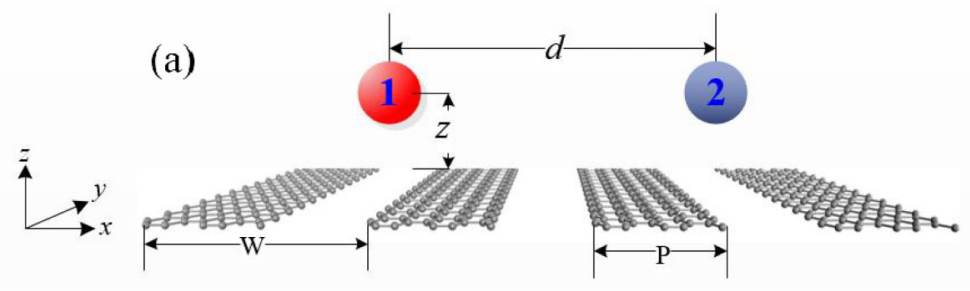

(b)

(c)
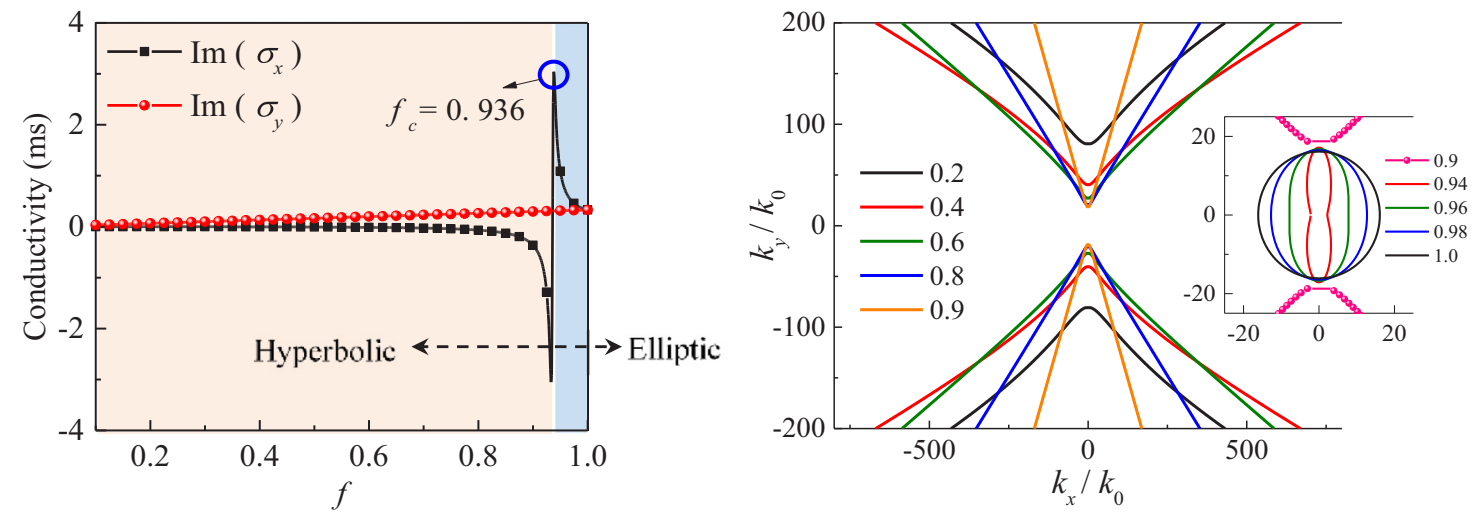

FIG. 2. (a) Schematic of NFRHT between two nanoparticles separated by an interparticle distance of $d$ above an array of GS, where $W$ and $P$ are the strip periodicity and ribbon width, respectively. $z$ is the particle-GS distance for the two nanoparticles. (b) The imaginary part of the optical conductivities along $x$ and $y$ directions with respect to the filling ratio. (c) The dispersion relations of the GS at a rotation angle of $\theta=0^{\circ}$ for different filling ratio in wave-vector space at $\omega_{r}=1.756 \times 10^{14} \mathrm{rad} / \mathrm{s}$.

decrease when the nanoparticles are in the very near field of the GS. Thus to guarantee the validity of the EMA for our calculations, the particle-GS distance $z$ should be several times greater than the strip periodicity. As detailed in Ref. [14], for $W=20 \mathrm{~nm}$, the EMA predicts the real heat flux well when $z \geqslant 60 \mathrm{~nm}$.

Let us begin by discussing the optical properties of GS at the $\omega_{r}$. As a concrete example, the parameter for the GS is chosen as a strip periodicity of $W=20 \mathrm{~nm}$ and a chemical potential of $\mu=0.5 \mathrm{eV}$. In Fig. 2(b), we show the imaginary parts of the conductivities $\operatorname{Im}\left(\sigma_{x}\right)$ and $\operatorname{Im}\left(\sigma_{y}\right)$ with respect to the filling ratio $f$. Meanwhile in Fig. 2(c), we plot equalfrequency curves (EFC) of the GS obtained from the dispersion relation [54] $2 k_{0}^{2} \eta\left(\sigma_{x x}+\sigma_{y y}\right)-2 \eta\left(k_{x}^{2} \sigma_{x x}+k_{y}^{2} \sigma_{y y}\right)+$ $k_{0} k_{z}\left(4+\eta^{2} \sigma_{x x} \sigma_{y y}\right)=0$, where $\eta$ is the free-space impedance. We see that at the resonance frequency of $\operatorname{SiC}, \operatorname{sgn}\left[\operatorname{Im}\left(\sigma_{x}\right)\right] \neq$ $\operatorname{sgn}\left[\operatorname{Im}\left(\sigma_{y}\right)\right]$ is achieved for a large range of $f$ lower than 0.936, indicating a hyperbolic topology in the EFC without limitation on the allowed values of $k_{x}$ and $k_{y}$, highlighting its unclosed contour that translates into ideally infinitely confined SPPs, i.e., infinite local density of states, propagating towards specific directions within the surface. However, we notice a topological transition point at $f=0.936$ beyond which $\operatorname{sgn}\left[\operatorname{Im}\left(\sigma_{x}\right)\right]=\operatorname{sgn}\left[\operatorname{Im}\left(\sigma_{y}\right)\right]$ is achieved providing an elliptic anisotropic topology in the EFC. Finally when $f=1$, the well-known elliptic isotropic topology for graphene is achieved. As shown in Fig. 2(b), The GS with a larger filling ratio having a larger contrast between $\operatorname{Im}\left(\sigma_{x}\right)$ and $\operatorname{Im}\left(\sigma_{y}\right)$ may favor SPPs propagation towards a specific direction, viz., the $y$ axis in the case. Based on Fig. 2(c), we can expect that the GS would have a large influence on the RHT between two nanoparticles.
We first discuss the conductance $h$ at $300 \mathrm{~K}$ between the two nanoparticles above the GS as a function of the filling ratio $f$ by fixing the particle-GS distance $z$ to the minimum value of $60 \mathrm{~nm}$ as shown in Fig. 3(a). The other parameters of GS are the same as those for Fig. 2. We consider a large interparticle distance $d=1 \mu \mathrm{m}$. Besides, we also illustrate the impact of the rotation of GS with respect to the reference coordinate system on the results.

We can see in Fig. 3(a) that the presence of the GS significantly modifies the RHT between the two nanoparticles. In most of the cases, the conductance is larger than that in the absence of the GS, which means that an enhancement of heat transfer is achieved, while is smaller than that in the presence of the graphene sheet. We stress that the enhancement of heat transfer is mainly due to the enhancement of the electromagnetic field on the GS with the excitation of anisotropic SPPs when the two nanoparticles are brought in proximity to the surface. The anisotropic SPPs propagate along the surface, and are coupled to the free-space propagating waves emitted by the nanoparticles, hence providing an additional channel to the energy transportation between the two nanoparticles. As for the NFRHT between two plates made from GS, due to the large wave vectors of the hyperbolic SPPs, the NFRHT are significantly enhanced in comparison with the case of two graphene sheets that support isotropic SPPs with limited wave vectors [14]. However, it is not the case in our configuration. When the graphene is patterned into an array of GS, a suppression of RHT is observed in most cases as depicted in Fig. 3(a). This can be understood as follows. For the configuration of two plates, the SPPs supporting large wave vectors generate a larger tunneling effect of evanescent waves, while the propagation capability along the surface is not important due to the 
(a)

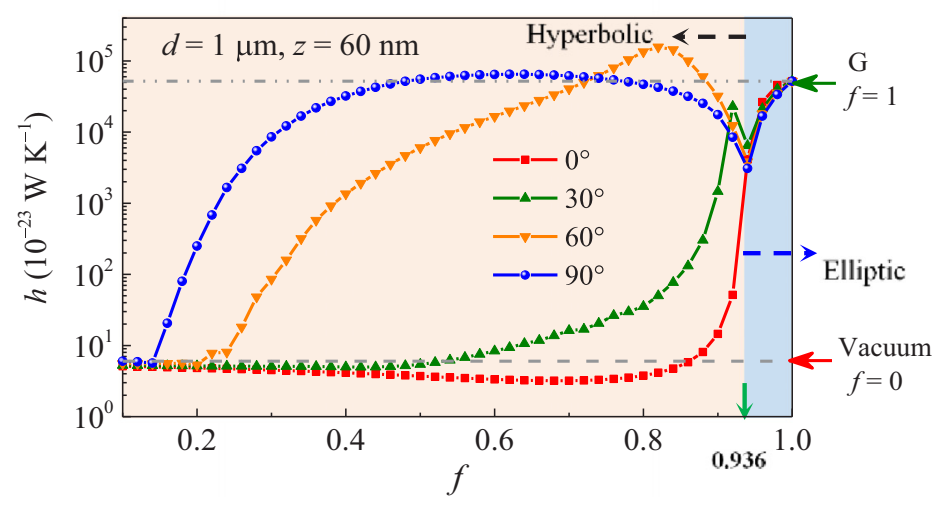

(b)

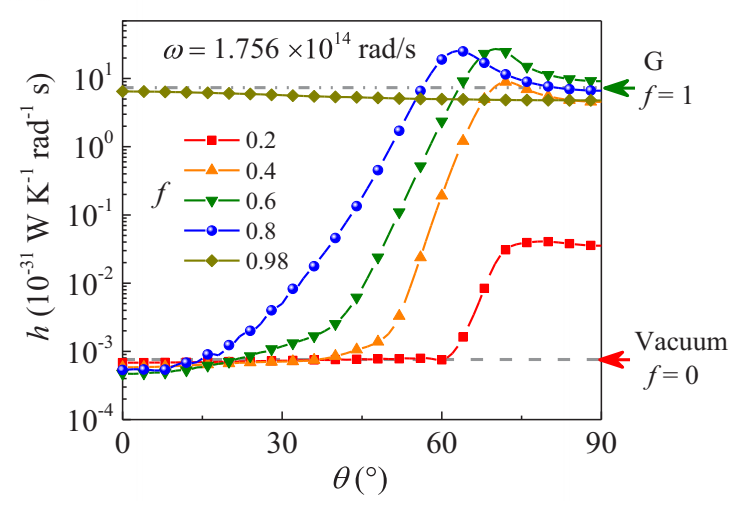

FIG. 3. (a) Total conductance as defined in Eq. (1) between two $\mathrm{SiC}$ nanoparticles in the presence of an array of GS at different rotation angles $\theta$ with respect to the filling ratio $f$. (b) Spectral conductance at $\omega_{r}$ with respect to the rotation angle $\theta$ for different filling ratios $f$. The two gray lines correspond to results in the case of graphene or vacuum. The particle-GS and interparticle distances are $z=60 \mathrm{~nm}$ and $d=1 \mu \mathrm{m}$, respectively. The chemical potential of the GS is fixed at $0.5 \mathrm{eV}$.

very large size of the surface. Nevertheless, the propagation characteristics play a large role in our configuration. This means that the propagation length, and the directionality of the SPPs would make a significant impact on the RHT between two nanoparticles. As the graphene sheet is patterned into strips, the isotropic waves are switched into anisotropic ones. Hence, although the SPPs support large wave vectors, they propagate towards specific angles, and at the same time are forbidden in other directions as shown in Fig. 2(c). Finally a lower conductance is revealed than that of a graphene sheet as shown in Fig. 3(a).

Now we discuss the results by setting the GS with different filling ratios at different angles with respect to the coordinates. We note that the RHT dominates at the resonant frequency of the $\mathrm{SiC}$ particle $\omega_{r}$ and no other resonance frequencies emerge in our configuration, thus the spectral conductance are able to predict the trend of the total conductance. Regarding this, we show the $h$ at $\omega_{r}$ with respect to the rotation angle in Fig. 3(b). We see in Figs. 3(a) and 3(b) that there are orders of magnitude differences for $h$ at different angles, highlighting the significant impact of the directionality of the anisotropic SPPs. It is easy to understand that the SPPs prefer to propagate along the graphene strips, viz., $y$ direction for $\theta=0^{\circ}$. Additionally, SPPs along the $x$ direction also exist, owing to the resonance coupling of the SPPs between the adjacent strips. Since the two nanoparticles are aligned with the $x$ axis, the SPPs along the $x$ direction have direct interactions with the RHT between two nanoparticles. In Fig. 2(c), as the filling ratio increases, the dispersion curve narrows, which means the allowed directions become extremely limited, exhibiting bidirectional SPPs. As a result, a suppression of heat transfer for the case of $\theta=0^{\circ}$ becomes prominent, and the conductance lowers further than the case without GS as observed in Fig. 3(a) over a large range of $f$. Nevertheless, by switching the angle to a larger one, the SPPs propagating towards the $x$ direction become more plentiful, hence an increase in $h$ is observed as shown in Fig. 3(b). A five-orders-of-magnitude enhancement between $90^{\circ}$ and $0^{\circ}$ at a filling ratio of 0.8 is observed. For $\theta>0^{\circ}$ in Fig. 3(a), we observe a monotonically increasing behavior over a large range of $f$. However, when the filling ratio increases sufficiently, the ratio between $\operatorname{Im}\left(\sigma_{x}\right)$ and $\operatorname{Im}\left(\sigma_{y}\right)$ becomes very large [see in Fig. 2(b)] while the SPPs are still hyperbolic when $f$ is below 0.936 . This means that the SPPs are extremely anisotropic along the GS, hence a decrease in $h$ is observed when $f$ is further increased. For the GS at a larger angle, the inflection point appears earlier. Interestingly, the $h$ for $0^{\circ}$ [see the curve colored in red as shown in Fig. 3(a)] increases significantly, switching the suppression effect to an enhancement effect of RHT when $f$ is above a certain value. As $f$ increases to a large value, the adjacent strips get very close, and hence the adjacent SPPs strongly couple with each other through tunneling effects. We can further observe an inflection point at $f=0.936$, which is consistent with the turning point as shown in Fig. 2(b) at which the hyperbolic topology becomes an elliptic anisotropic topology in the EFC [see in Fig. 2(c)]. After this value for $f$, elliptic anisotropic SPPs become more similar to the isotropic case. $h$ thus rises for the GS at any angle, and not surprisingly, the results converge to those in the presence of the graphene sheet as the filling ratio goes to 1.0 .

To give an intuitive explanation of the underlying physics, we plot two kinds of contours in the wave-vector space and spatial space in Figs. 4(a) and 4(b), respectively. The frequency is chosen to be resonant, viz., $\omega_{r}=1.756 \times$ $10^{14} \mathrm{rad} / \mathrm{s}$. Figure 4(a) shows the real part of the first component of the reflected Green's function for the graphene and GS at an angle of $0^{\circ}, 30^{\circ}, 60^{\circ}$, and $90^{\circ}$. We can observe that the isotropic as well as the anisotropic SPPs at different angles are all excited exactly at the dispersion curves, viz., at the resonance wave vectors. The transitions of the propagation characteristics of the evanescent waves from graphene to GS and from $0^{\circ}$ to $90^{\circ}$ of GS are also clearly illustrated, confirming our qualitative discussions above. Figure 4(b) displays the spatial distributions of the radiated electric field energy density $\mu_{e}(\mathbf{r}, \omega)=\frac{2 \varepsilon_{0}^{2}}{\pi \omega} \sum_{j} \chi_{j} \Theta\left(\omega, T_{j}\right) \operatorname{Tr}\left[\mathbb{Q}_{r j} \mathbb{Q}_{r j}^{*}\right]$, where $\mathbb{Q}_{r j}=$ $\omega^{2} \mu_{0}\left(\mathrm{G}_{0}^{r j}+\mathrm{G}_{R}^{r j}\right) \mathcal{G}$ and $\Theta\left(\omega, T_{j}\right)=\hbar \omega n(\omega, T)$ [28] is the mean energy of the Planck oscillator at temperature $T$, in 


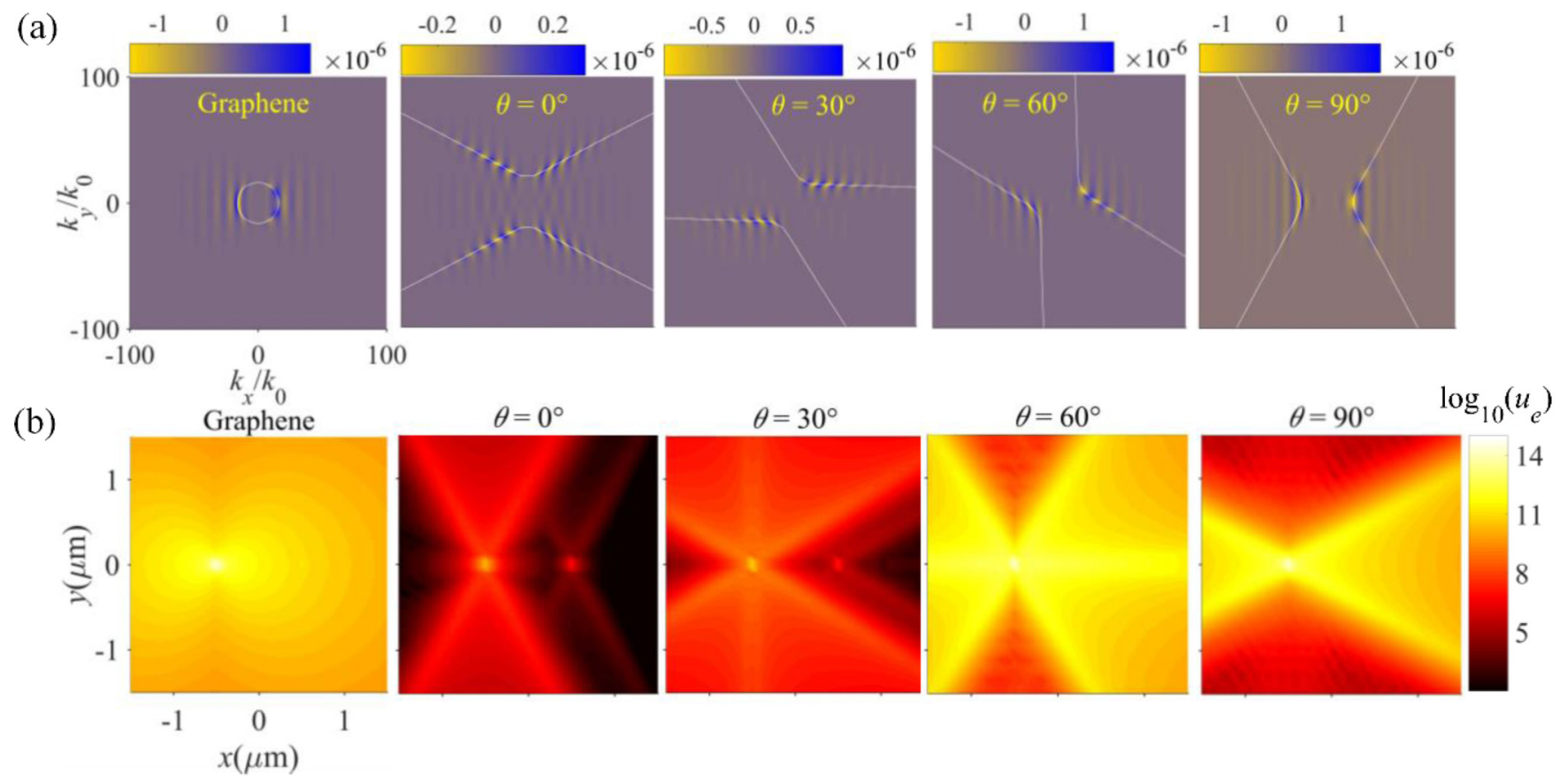

FIG. 4. (a) Wave-vector contours of the real part of the first component of the reflected Green's function $\operatorname{Re}\left[\mathbf{G}_{R, E E}(1,1)\right]$. (b) Spatial contours of the electric field energy density $u_{e}$ at $z=30 \mathrm{~nm}$ for the graphene sheet and the GS rotated at different angles. In panel (a), the white line corresponds to the equal-frequency curves. For panel (b), the temperatures of the left and right nanoparticles are kept at 300 and $0.5 \mathrm{~K}$, respectively. The interparticle and particle-GS distances are $d=1 \mu \mathrm{m}$ and $z=60 \mathrm{~nm}$, respectively. The frequency is fixed at $\omega_{r}=1.756 \times 10^{14} \mathrm{rad} / \mathrm{s}$. The filling ratio and chemical potential of the GS are 0.8 and $0.5 \mathrm{eV}$, respectively.

the plane $z=30 \mathrm{~nm}$. We see that the presence of the GS significantly modifies the energy distributions in the physical space, which exhibit distinctive inhomogeneity. However, the case with a graphene sheet is much more homogeneous. An inspection of the plots at $0^{\circ}$ and $90^{\circ}$ clearly illustrates that a higher energy density prefers to be distributed along the GS. In Fig. 4(a), for small angles of $\theta=0^{\circ}$ or $30^{\circ}$, we see that the EFC and the line $k_{y}=0$ do not cross each other, thus few SPPs directly propagate along the $x$ direction. While the EFC and $k_{y}=0$ cross each other for $\theta=60^{\circ}$ and $90^{\circ}$, indicating the excitation of the SPPs along the $x$ axis, a direct propagation channel at the $x$ axis is established. We thus observe a large energy density distributed along the $x$ axis especially for $\theta=60^{\circ}$ as shown in Fig. 4(b). These physics arguments could give an interpretation for the climbing trend of the blue line in Fig. 3(b).

Interestingly, as depicted in Figs. 3(a) and 3(b), we see that although the allowed directions for the GS are limited, the conductance of GS can be even larger than that of the graphene and the GS at $\theta=90^{\circ}$ when the GS is rotated at a proper angle, i.e., $60^{\circ}$ for $f=0.8$ or $70^{\circ}$ for $f=0.6$ [see in Fig. 3(b)]. This surprising result is a clear indication of the complexities and richness of heat transport in our system. For $f=0.8$, this enhancement of RHT is mainly reliant on the fact that the intersection point for $\theta=60^{\circ}$ is located at a larger wave vector than the one for the graphene sheet or the GS at $\theta=90^{\circ}$ as shown in Fig. 4(a), hence the propagation of SPPs along $x$ axis carrying a very large wave vector is achieved. Nevertheless, due to the weak coupling SPPs between adjacent GS with a small filling ratio, this enhancement of RHT cannot be achieved at any rotation angle, i.e., $f=0.2$ in Fig. 3(b).

\section{DISTANCE DEPENDENCE OF THE HEAT TRANSFER}

We now address the question of the dependence of the total conductance regulation on the distances. We stress that there are two distances in our configuration, viz., the interparticle distance $d$ and the particle-surface distance $z$. It is expected that the first distance $d$ is a relevant parameter to highlight the propagation characteristics of the anisotropic SPPs along the surface. Since the surface waves are evanescent waves with amplitudes decreasing away from the interface on a scale of one wavelength, we thus expect that the dependence of the second distance $z$ could be used to elucidate the tunneling effects in our configuration.

The results of conductance with respect to the first distance $d$ are presented in Fig. 5(a). In the inset, we plot the amplification factor $h / h_{0}$ (where $h_{0}$ is the conductance between the two nanoparticles without the GS) as a function of $d$. Equations (1) and (5) imply that the behavior of the conductance in vacuum at small distances is $d^{-6}$ [29]. We thus see that the conductance decreases quickly in the absence of the GS. In the presence of GS at a small angle, since the transportation of the evanescent wave along the $x$ direction is blocked, the reflected energy provided by GS is suppressed. Hence, minor differences between the curve with GS at $0^{\circ}$ and the one in vacuum are observed. For the GS at a larger angle, although the conductance decreases monotonically, the decay rate is lower than without GS. Thus, we see a significant 

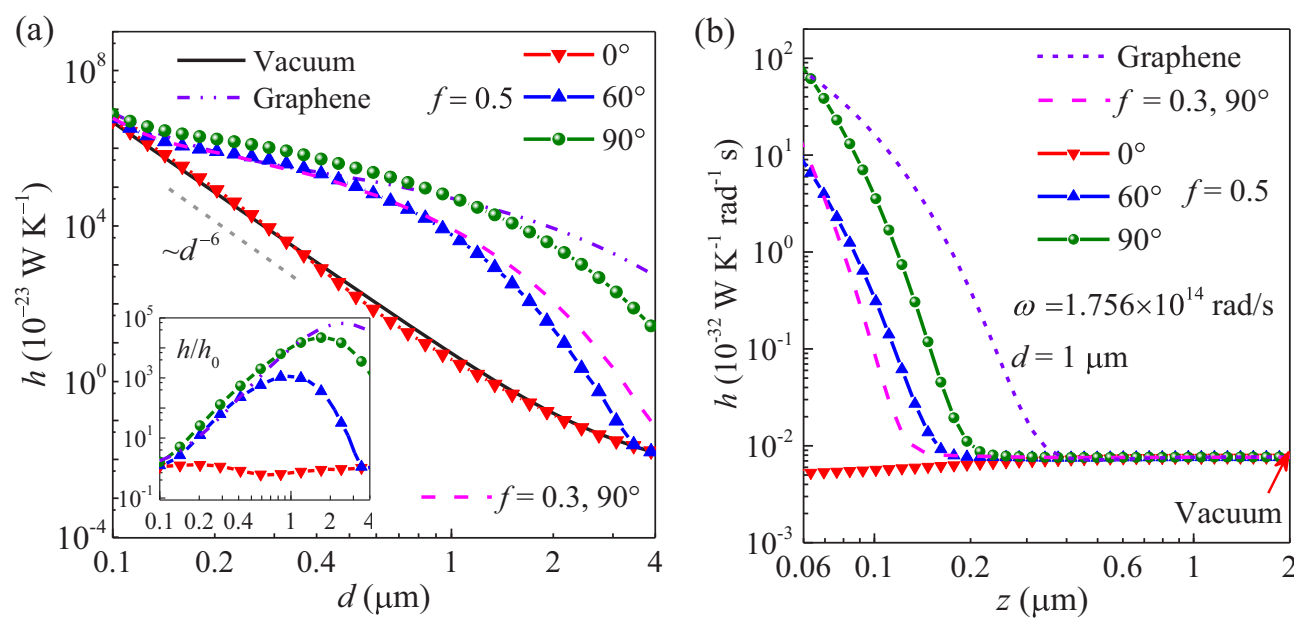

FIG. 5. (a) Total conductance $h$ with respect to $d$ between two SiC nanoparticles placed at distance $z=60 \mathrm{~nm}$ from an array of GS. The inset shows the ratio $h / h_{0}$ between the conductances in the presence and absence of the surface as a function of $d$. (b) Spectral conductance at $\omega_{r}$ with respect to the particle-surface distance for GS rotated at different angles. The chemical potential of the GS is $0.5 \mathrm{eV}$.

enhancement of RHT between the two nanoparticles at a large range of interparticle distances as depicted in the inset of Fig. 5(a). The amplification increases monotonically and remarkably reaches a value around $10^{4}$ for $90^{\circ}$ at $d=1.74 \mu \mathrm{m}$ and 400 for $60^{\circ}$ at $d=0.92 \mu \mathrm{m}$, and then decreases. We stress that this dependence with respect to $d$ is mainly attributed to the propagation length of the SPPs [2], viz., $L=1 / \operatorname{Im}(K)$, which can be comparable to one or several wavelengths. $K$ is the resonant parallel wave vector, determined by the dispersion relations of the surface. Based on the physical meaning of $L$, we can thus expect that in the range of $d<L$, the SPPs excited by the first particle could propagate to the position below the second particle with a big amplitude, subsequently tunneling into it. As for a suspended graphene sheet, $K$ equals $\sqrt{k_{0}^{2}-\left(2 \varepsilon_{0} \omega / \sigma\right)^{2}}$, implying a propagation length of $1.85 \mu \mathrm{m}$ towards all the directions along the surface at $\omega_{r}$. We thus observe a decreasing trend in the amplification curve after $d \approx$ $2 \mu \mathrm{m}$. However, we can expect that since the supported SPPs are anisotropic, the propagation length of the SPPs supported by the GS varies with the directions. We find $L$ by calculating the dispersion relations of the GS. The results at $\omega_{r}$ for the GS rotated at $90^{\circ}$ are presented in Fig. 6(a). Not surprisingly, we see that the propagation length is valid only at a limited range of directions. Meanwhile, we observe that $L$ is largest towards the graphene strip and monotonically decreases with respect to the rotation angle. The effective propagate length of GS is thus shorter than that of graphene. Hence we see in Fig. 5(a) the amplification curve starts to decrease at a lower value of $d$ than that of graphene. For the GS rotated at an angle less than $90^{\circ}$, due to a shorter propagation length along $x$ axis, the curve also decreases at a shorter distance as shown in Fig. 5(a). Furthermore, Fig. 6(a) shows that for the GS with a larger filling factor, the $L$ curve exhibits a higher value. We thus see in Fig. 5(a) that the total conductance for $f=0.3$ decreases faster than that of $f=0.5$ at the same angle of $90^{\circ}$.

Moreover, we observe in Fig. 5(a) a larger conductance at a small interparticle distance in the presence of GS with $f=0.5$ than the one in the presence of graphene. This amplification of the flux has also been noticed for $f=0.8$ as shown in Fig. 3. We stress that this amplification of the flux could be achieved at a large range of interparticle distances once the rotation angles and filling ratios were tuned properly. In order to confirm this point, we show in Fig. 7 the ratio between the spectral conductance at $\omega_{r}$ of the configuration in the presence of GS and the one in the presence of graphene as a function of $f$ with respect to the rotation angle of GS. Note that the white region in Fig. 7 separates the amplification and suppression regions which correspond to the red and blue colors, respectively. The amplification of flux can be therefore achieved at short, medium, and long distances. The filling ratios of the GS satisfying the amplification are below 0.9, indicating hyperbolic anisotropic SPPs along the GS [see in Fig. 2(a)]. This enhancement of the GS over the graphene sheet implies the hyperbolic anisotropic SPPs' superiority over the elliptic or isotropic ones. At a short distance of $150 \mathrm{~nm}$ depicted in Fig. 7(a), the higher amplification factor prefers the GS rotated at a larger angle, especially reaching a maximum value at $\theta=90^{\circ}$. However, at a larger distance of $500 \mathrm{~nm}$ or $1 \mu \mathrm{m}$ as shown in Figs. 7(b) and 7(c), the maximum amplification factor increases to a larger value and is achieved at a smaller rotation angle. Moreover, we see that the green dotted line shows a descending trend as a function of $f$, which means that a maximum ratio is achieved at a smaller angle for a larger filling ratio. This is because, for a larger filling ratio, the opening angle of the dispersion relations is much smaller as shown in Fig. 2(c), hence the intersection between the dispersion curves and $x$ axis could be accomplished at small angles.

Now we turn our attention to elucidate the effect of the second distance $z$. Figure 5(b) shows the spectral conductance at $\omega_{r}$. We see that an enhancement of three orders of magnitude is achieved in the near field. As $z$ increases from near field to far, due to the decreasing amplitude of the tunneling evanescent waves, the heat fluxes converge to the one in the absence of the surface. The enhancement or suppression of heat transfer provided by the surface are negligible in the far field. To give an explanation on the above phenomenon, we 
(a)

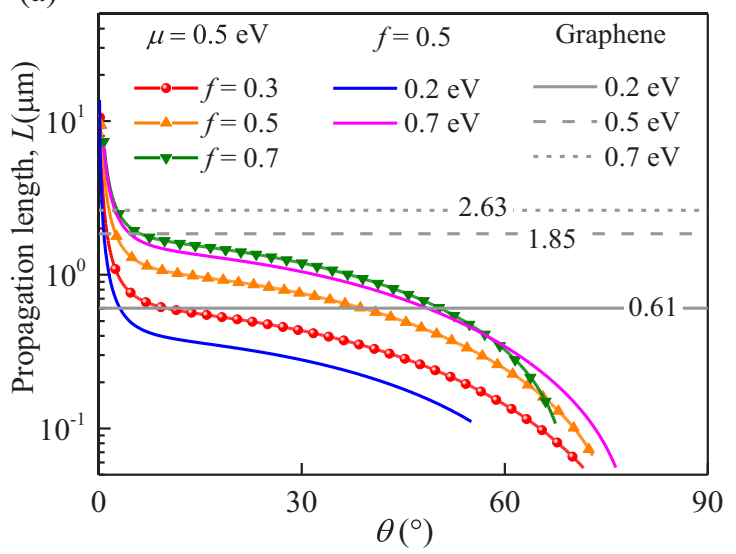

(b)

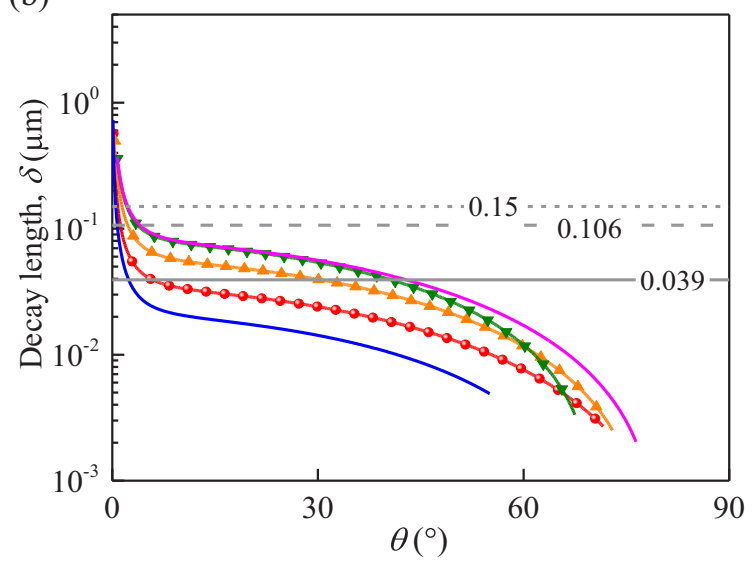

FIG. 6. The properties of the SPPs supported on the GS. (a) Propagation length of the SPPs along the surface at $\omega_{r}$ calculated from $L=1 / \operatorname{Im}(K)$. The gray lines denote the $L$ for the graphene sheet. (b) Decay length of the SPPs along the $z$ direction at $\omega_{r}$ calculated from $\delta=1 / \operatorname{Im}\left(k_{z}\right)$. The gray lines denote the $\delta$ for the graphene sheet.

plot in Fig. 6(b) the SPPs decay length $\delta=1 / \operatorname{Im}\left(k_{z}\right)$ at $\omega_{r}$ [2] in the direction perpendicular to the surface in the vacuum. We see that the decay length curves exhibit the same trends as those of the propagation lengths in Fig. 6(a). The SPPs along the graphene strip possess the longest decay length. We thus see that the heat flux of the GS rotated at $60^{\circ}$ converges to a constant value at a shorter $z$ than that of $90^{\circ}$ as shown in Fig. 5(b). Due to the decreasing trend of the decay length of the SPPs for the GS, the effective $\delta$ of GS is lower than that in the presence of graphene. A faster decay rate in the presence of the GS than that of the graphene is observed in the heat flux curve as shown in Fig. 5(b).

Since several similar investigations of the RHT between nanoparticles in the presence of a semi-infinite substrate have been published recently [28-30], it is therefore necessary to compare those with the present study. The conductance with respect to the interparticle distance in the presence of a flat graphene, the $\mathrm{GS}\left(\theta=68^{\circ}, f=0.7\right)$, a semi-infinite $\mathrm{SiC}$ substrate, and a graphene-covered $\mathrm{SiC}$ substrate are plotted in Fig. 8(a). The particle-surface distance is fixed at $60 \mathrm{~nm}$. We see that the presence of the suspended GS or a graphene sheet predicts larger conductances than those of a semi-infinite $\mathrm{SiC}$ substrate with or without the graphene sheet on top. The conductance in the presence of a graphene-covered $\mathrm{SiC}$ substrate is lower than that of the uncovered one. Moreover, an enhancement of RHT over graphene is observed when the graphene is patterned into an array of graphene strips with a filling ratio of $f=0.7$ rotated at an angle of $\theta=68^{\circ}$, which is mainly attributed to the propagation of SPPs along the $x$ axis with a larger wave vector for the GS.

For the sake of understanding these phenomena, we plot the spectral conductance at an interparticle distance $1 \mu \mathrm{m}$ in Fig. 8(b). Note that the pink line obtained by our program for the anisotropic metasurface coincides with the square dots from the framework for the isotropic slab in Ref. [29]. This confirms the prediction obtained in the paper on a single graphene sheet. Since the SPPs supported on the suspended GS or graphene cover a wide frequency range and extend to a large wave vector, we thus observe that their spectral conductances are much larger than the others in the

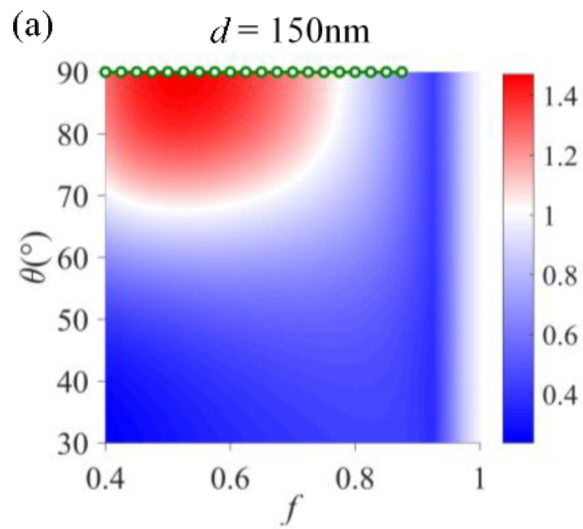

(b) $d=500 \mathrm{~nm}$

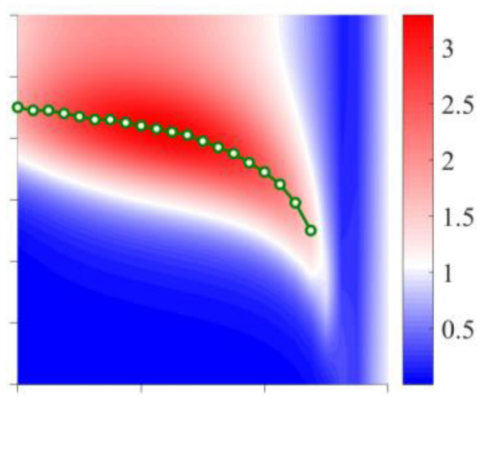

(c) $\quad d=1 \mu \mathrm{m}$

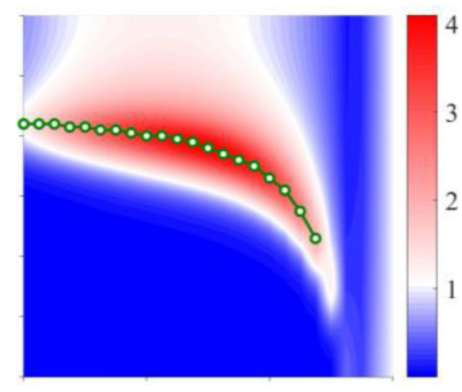

FIG. 7. The ratio between the spectral conductance at $\omega_{r}$ of the configuration in the presence of GS and the one in the presence of graphene as a function of the filling ratio for the GS with respect to the rotation angle of the GS at an interparticle distance of (a) $150 \mathrm{~nm}$, (b) $500 \mathrm{~nm}$, and (c) $1 \mu \mathrm{m}$. The green lines with open circles correspond to the maximum ratio. The chemical potential and the particle-GS distances are $0.5 \mathrm{eV}$ and $60 \mathrm{~nm}$, respectively. 

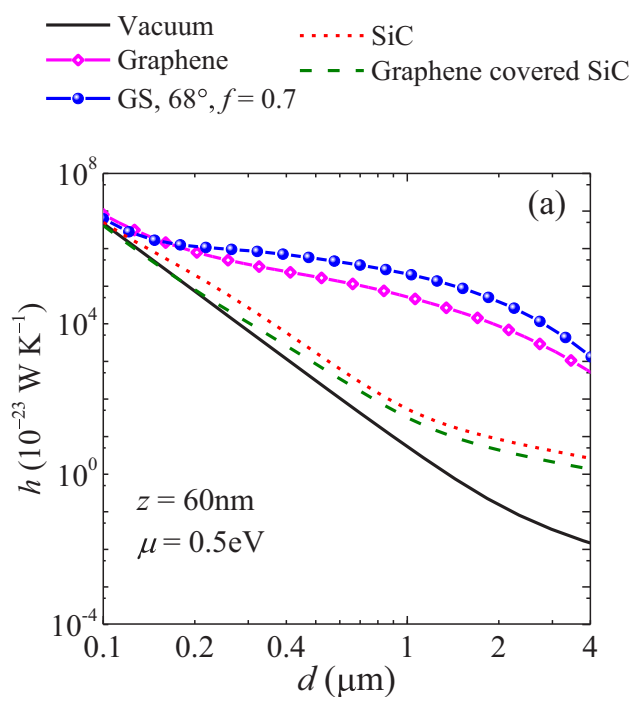

$$
\begin{aligned}
& - \text { Vacuum } \\
& \longrightarrow-\mathrm{GS}, 68^{\circ}, f=0.7 \quad \square \quad \text { Graphene, Ref. [29] } \\
& -\nabla-\mathrm{SiC} \\
& \square-\text { Graphene, } f=1.0
\end{aligned}
$$

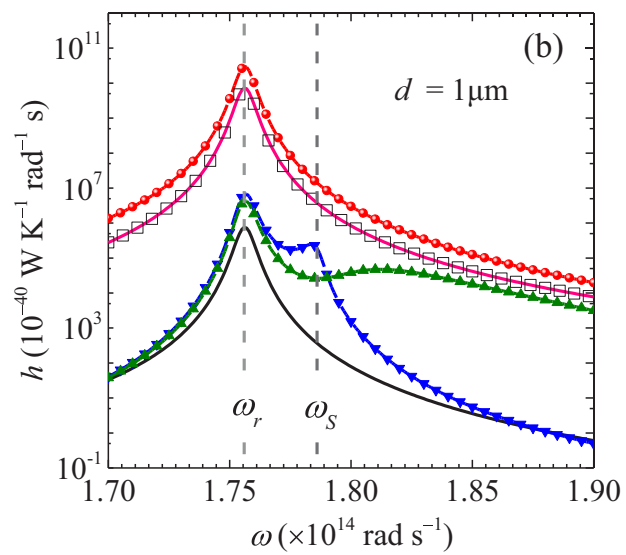

FIG. 8. Comparison study of the RHT between two SiC nanoparticles in the presence of a graphene sheet, the GS $\left(\theta=68^{\circ}, f=0.7\right)$, a semi-infinite $\mathrm{SiC}$ substrate and a graphene-covered $\mathrm{SiC}$ substrate, respectively. (a) Total conductance $h$ as defined in Eq. (1) with respect to the interparticle distance $d$. (b) Spectral conductance at an interparticle distance $1 \mu \mathrm{m}$. The two vertical dashed lines in panel (b) denote the resonance frequencies of the nanoparticle and the $\mathrm{SiC}$-vacuum interface, respectively. The chemical potential of the GS or the graphene sheet is $0.5 \mathrm{eV}$. The particle-surface distance is $z=60 \mathrm{~nm}$.

whole frequency range under consideration, hence larger total conductances are observed in Fig. 8(a). Note that the dispersion relations show no horizontal frequency asymptote in the frequency-wave-vector plane for the suspended GS and the graphene sheet, thus no additional resonant peaks emerge in the spectral plots. However, this is not the case in the presence of the $\mathrm{SiC}$ substrate. As discussed in Refs. [28-30], since the SPhPs of the SiC-vacuum interface exhibit resonance at $\omega_{s}=1.786 \times 10^{14} \mathrm{rad} / \mathrm{s}$ corresponding asymptotically to the condition $\varepsilon(\omega)+2=0$, we observe an additional peak at $\omega_{s}$ on the spectral curve in Fig. 8(b). As a graphene sheet is deposited on the $\mathrm{SiC}$, the SPPs supported by graphene alone couples with the SPhPs of the SiC-vacuum interface, producing hybrid modes whose dispersion relations bend toward a higher frequency but a lower wave vector compared to the SiC-vacuum SPhPs alone. We thus see that the right spectral peak shifts to a larger frequency in Fig. 8(b) and the conductance is lower than the uncovered $\mathrm{SiC}$ as shown in Fig. 8(a). We note that the SPhPs of the SiC possess a high lateral wave vector at $\omega_{s}$, but $\omega_{s}$ does not match with the particle resonance frequency $\omega_{r}$ and the lateral wave vector of the $\mathrm{SPhPs}$ at $\omega_{r}$ is very low. We thus observe that the spectral peaks of the graphene-covered or uncovered $\mathrm{SiC}$ are lower than those of the suspended GS or graphene sheet, hence lower total conductances are observed in Fig. 8(a). However, things would be different at a large interparticle distance. We find that the propagation length of the SPhPs supported on the $\mathrm{SiC}$-vacuum interface is about $20 \mu \mathrm{m}$ at the nanoparticle resonance frequency [28], which is much larger than the $1.85-\mu \mathrm{m}$ value of the graphene sheet depicted in Fig. 6(a). Hence, it is expected that the enhancement of RHT in the presence of $\mathrm{SiC}$ over the vacuum would be more significant at a long distance than those of the GS or graphene sheet. Based on this comparison study and the discussion in Sec. III, we remark that in addition to offering a way to modulate the RHT the anisotropic GS could also provide a larger enhancement at the medium interparticle distance than the isotropic $\mathrm{SiC}$ substrate.

\section{EFFECT OF THE CHEMICAL POTENTIAL}

The chemical potential $\mu$ represents an adjustable parameter allowing us to actively tune the optical properties of graphene. We now examine the influence of the chemical potential on the RHT between nanoparticles. The variation of $\mu$ with $d=1 \mu \mathrm{m}, z=60 \mathrm{~nm}$, and $f=0.5$ is shown in Fig. 9(a). We also present in Fig. 9(b) the dispersion relations $\operatorname{Re}(K) / k_{0}$ and propagation length $L$ for the graphene, and in Fig. 9(c) the dispersion relations for the GS. We see that the impact of the surface on the RHT between nanoparticles is negligible at a very low chemical potential, which results from the near-zero propagation length of the SPPs in graphene though an increasing wave vector as shown in Fig. 9(b). We expect that the propagation length for the GS also increases monotonically with respect to the chemical potential, but at a smaller rising rate than that of graphene. The impact of GS thus appears at a larger chemical potential as depicted in Fig. 9(a). We further observe that for the graphene after the impact emerges, with an increase in $\mu$ the curve climbs rapidly and reaches a maximum after which it declines. The climbing trend is a result of the increasing propagation length as well as a large enough wave vector of the SPPs as depicted in Fig. 9(b). However, we expect that the decline of the wave vector would reduce the heat flux. As the chemical potential increases further, this reduction of RHT offsets or even exceeds the increment of RHT contributing from the large propagation length, hence a declining trend is observed in the black curve. As for the GS, although the $k_{x}$ of the equal frequency curve moves to a lower value as $\mu$ increases, it opens and extends to infinity as depicted in Fig. 9(c). As a 
(a)

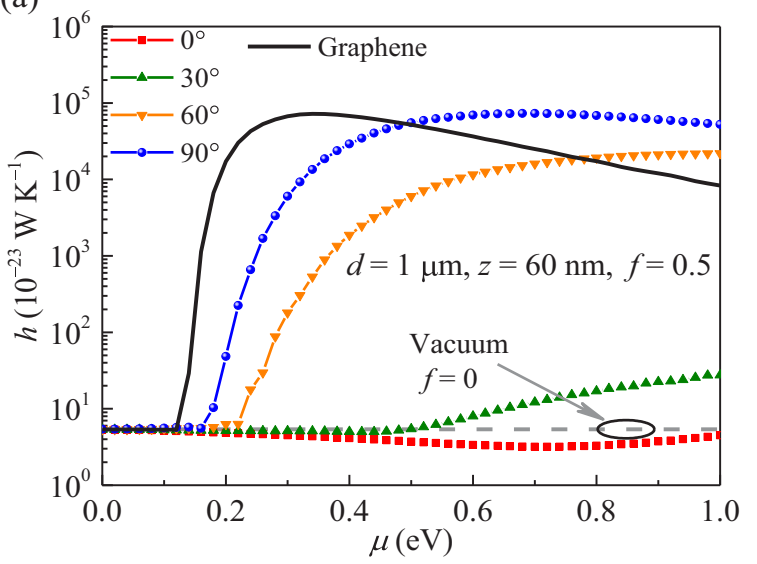

(b)

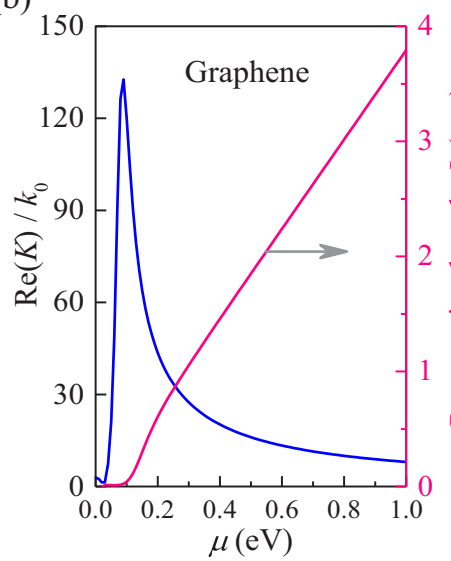

(c)

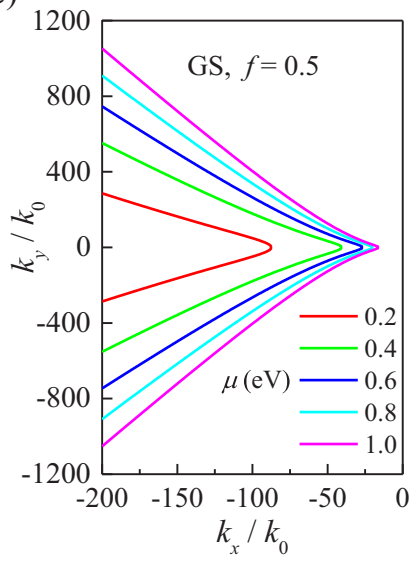

FIG. 9. (a) Total conductance between the two nanoparticles with respect to the chemical potential of the graphene and GS. (b) The lateral wave vector and propagation length of the graphene sheet. (c) The dispersion relations at $k_{x}<0$ for the GS. The parameters are kept as $d=1 \mu \mathrm{m}, z=60 \mathrm{~nm}$, and $f=0.5$.

result, for the GS, the reduction rate of RHT resulting from the decreasing in $k_{x}$ is lower than that of the graphene. We thus see in Fig. 9(a) that the curve for the GS rotated at $90^{\circ}$ is above that of the graphene at a large chemical potential. More specifically at $1.0 \mathrm{eV}$ in Fig. 9(a), the conductance for the GS is six times the value for the graphene. Based on this, we can conclude that for a large chemical potential, the GS is more preferable to amplify the conductance between two nanoparticles to a large value than the unpatterned graphene. As for the GS rotated at $0^{\circ}$, since the propagation of the SPPs along the $x$ axis is forbidden, the increasing propagation length makes no contribution to the RHT. Thus due to the decrease in $k_{x}$, the suppression of the heat flux gets more dominated at a larger chemical potential.

\section{CONCLUSIONS}

This paper has presented the modification of RHT between two dielectric $(\mathrm{SiC})$ nanoparticles when placed in the proximity of an array of GS supporting anisotropic SPPs. The SPPs topology of the GS used could be tuned from isotropic to hyperbolic. This paper has demonstrated that in our configuration, due to the excitation and propagation of the anisotropic surface waves, the ambient-temperature conductance between the nanoparticles could be amplified, and regulated over several orders of magnitude. The dependence of conductance on the filling factor and the rotation angle of the GS have been analyzed. The underlying physics are interpreted qualitatively by analyzing the distribution of the reflected Green's function in the wave-vector space as well as the spatial electric density distribution, clearly highlighting the role played by the anisotropic surface mode. The dependence of the conductance regulation has been presented for two distances, interparticle and particle-surface distances. The enhancement of RHT was observed to be significant at a lateral distance comparable to the propagation length of the SPPs. Interestingly, we have also observed that if the rotation angle and filling ratio are tuned into proper ones, the conductance in the presence GS could be larger than the one in the presence of the graphene sheet at short, medium, and long interparticle distances. This surprising result is a clear indication of the complexity and richness of heat transport in this system. In addition, the amplification effect has been demonstrated to be lost at different large vertical distances for the GS rotated at different angles, as expected since surface waves are confined in the vicinity of the surface. The decay length of the SPPs was used to explain quantitatively these angle dependences. Furthermore, the chemical potential has been shown to dramatically modify and allow tuning of the RHT. Due to the hyperbolic topology of the SPPs supported on the GS, at a large chemical potential, the GS is more preferable to amplify the RHT between two nanoparticles to a large value than the unpatterned graphene.

So far, we have numerically investigated the NFRHT between two nanoparticles in the proximity of the GS. However, it is necessary to discuss the possible experimental realization of our configuration. The system discussed in this work consists of the particle and surface, so we can draw the experience from NFRHT measurements between sphere and flat plate, which are well studied configurations [4,5559]. For instance, Gang et al. [4,55] and Rousseau et al. [56] separately developed sensitive techniques to measure NFRHT between a microsphere and a substrate using a bimaterial atomic force microscope (AFM) cantilever. Based on the same experimental idea one may think to develop an experimental setup for the configuration we have studied. The two nanoparticles could be attached separately to the tips of bimaterial AFM cantilevers with ultraviolet adhesive. The base of the cantilever, the metasurface, particle 2, and the rest of the apparatus are approximately at the ambient temperature. We then give particle 1 a tiny temperature increment. The bimaterial cantilever for particle 2 bends as a result of a temperature increase, caused by heat transfer between the two particles. This bending signal is detected interferometrically using a laser that is focused on the rear side of the cantilever and then is converted into heat transfer-distance curves.

This work represents a step in the study of the modification of energy exchanges mediated by an anisotropic surface and is expected to provide a more powerful way to regulate the 
energy transport in the particle systems than that by an isotropic surface, and facilitates a way to explore the anisotropic optical properties of the metasurface based on the measured heat transfer properties. This study could be naturally extended to examine the RHT of a chain of nanoparticles or nanoparticle clusters mediated by the anisotropic surface, and also the RHT in nonreciprocal dispersive systems $[60,61]$ where unidirectional heat transfer becomes possible [62]. In addition, a similar study could be performed for the nanoparticles placed at each side of an anisotropic planar slab where the transmission model is needed. Note that if we consider the problem of heat exchange between two particles fixed at different temperatures, the temperature gradient would emerge on the GS, which means that a model considering the energy budget and phonon heat transport needs to be proposed.

\section{ACKNOWLEDGMENTS}

We thank J. S. Gomez-Diaz and J. Dong for valuable discussions. This work was supported by the National Natural Science Foundation of China (Grant No. 51706053), as well as the Fundamental Research Funds for the Central Universities (Grant No. HIT. NSRIF. 201842), and by the China Postdoctoral Science Foundation (Grant No. 2017M610208). M. A. acknowledges support from the Institute Universitaire de France, Paris, France (UE).
[1] D. Polder and M. Van Hove, Phys. Rev. B 4, 3303 (1971).

[2] K. Joulain, J. P. Mulet, F. Marquier, R. Carminati, and J. J. Greffet, Surf. Sci. Rep. 57, 59 (2005).

[3] S. Basu, Z. M. Zhang, and C. J. Fu, Int. J. Energy Res. 33, 1203 (2009).

[4] S. Shen, A. Narayanaswamy, and G. Chen, Nano Lett. 9, 2909 (2009).

[5] K. Kim, B. Song, V. Fernandez-Hurtado, W. Lee, W. Jeong, L. Cui, D. Thompson, J. Feist, M. T. Homer Reid F. J. Garcia-Vidal, J. C. Cuevas, E. Meyhofer, and P. Reddy, Nature (London) 528, 387 (2015).

[6] K. Ito, A. Miura, H. Iizuka, and H. Toshiyoshi, Appl. Phys. Lett. 106, 083504 (2015).

[7] M. P. Bernardi, D. Milovich, and M. Francoeur, Nat. Commun. 7, 12900 (2016).

[8] A. Kittel, W. Muller-Hirsch, J. Parisi, S.-A. Biehs, D. Reddig, and M. Holthaus, Phys. Rev. Lett. 95, 224301 (2005).

[9] Y. De Wilde, F. Formanek, R. Carminati, B. Gralak, P.-A. Lemoine, K. Joulain, J.-P. Mulet, Y. Chen, and J.-J. Greffet, Nature (London) 444, 740 (2006).

[10] R. Messina and P. Ben-Abdallah, Sci. Rep. 3, 1383 (2013).

[11] C. R. Otey, W. T. Lau, and S. Fan, Phys. Rev. Lett. 104, 154301 (2010).

[12] P. Ben-Abdallah and S. A. Biehs, Phys. Rev. Lett. 112, 044301 (2014).

[13] X. L. Liu, R. Z. Zhang, and Z. M. Zhang, ACS Photon. 1, 785 (2014).

[14] X. L. Liu and Z. M. Zhang, Appl. Phys. Lett. 107, 143114 (2015).

[15] Y. Yang and L. Wang, Phys. Rev. Lett. 117, 044301 (2016).

[16] K. Shi, F. Bao, and S. He, ACS Photon. 4, 971 (2017).

[17] B. Zhao, B. Guizal, Z. M. M. Zhang, S. H. Fan, and M. Antezza, Phys. Rev. B 95, 245437 (2017).

[18] P. Doyeux, S. A. H. Gangaraj, G. W. Hanson, and M. Antezza, Phys. Rev. Lett. 119, 173901 (2017).

[19] H. Iizuka and S. Fan, Phys. Rev. Lett. 120, 063901 (2018).

[20] P. Ben-Abdallah, K. Joulain, J. Drevillon, and C. Le Goff, Phys. Rev. B 77, 075417 (2008).

[21] P. Ben-Abdallah, S.-A. Biehs, and K. Joulain, Phys. Rev. Lett. 107, 114301 (2011).

[22] P. Ben-Abdallah, R. Messina, S.-A. Biehs, M. Tschikin, K. Joulain, and C. Henkel, Phys. Rev. Lett. 111, 174301 (2013).
[23] V. Yannopapas and N. V. Vitanov, Phys. Rev. Lett. 110, 044302 (2013).

[24] J. Dong, J. M. Zhao, and L. H. Liu, J. Quantum Spectrosc. Radiat. Transf. 197, 114 (2017).

[25] J. Dong, J. Zhao, and L. Liu, Phys. Rev. B 95, 125411 (2017).

[26] K. Asheichyk, B. Müller, and M. Krüger, Phys. Rev. B 96, 155402 (2017).

[27] M. G. Luo, J. Dong, J. M. Zhao, L. H. Liu, and M. Antezza, Phys. Rev. B 99, 134207 (2019).

[28] J. Dong, J. Zhao, and L. Liu, Phys. Rev. B 97, 075422 (2018).

[29] R. Messina, S.-A. Biehs, and P. Ben-Abdallah, Phys. Rev. B 97, 165437 (2018).

[30] K. Asheichyk and M. Kruger, Phys. Rev. B 98, 195401 (2018).

[31] A. Poddubny, I. Iorsh, P. Belov, and Y. Kivshar, Nat. Photon. 7, 948 (2013)

[32] Y. Guo and Z. Jacob, Opt. Express 21, 15014 (2013).

[33] I. S. Nefedov, A. J. Viitanen, and S. A. Tretyakov, Phys. Rev. E 71, 046612 (2005).

[34] M. Silveirinha and N. Engheta, Phys. Rev. Lett. 97, 157403 (2006).

[35] J. Gomis-Bresco, D. Artigas, and L. Torner, Nat. Photon. 11, 232 (2017).

[36] C. Yan, K. Y. Yang, and O. J. F. Martin, Light Sci. Appl. 6, e17017 (2017).

[37] H. C. Chu, Q. Li, B. B. Liu, J. Luo, S. L. Sun, Z. H. Hang, L. Zhou, and Y. Lai, Light Sci. Appl. 7, 50 (2018).

[38] Y. B. Zhu, Z. Y. Li, Z. Hao, C. Dimarco, P. Maturavongsadit, Y. F. Hao, M. Lu, A. Stein, Q. Wang, J. Hone, N. F. Yu, and Q. Lin, Light Sci. Appl. 7, 67 (2018).

[39] M. Papaioannou, E. Plum, E. T. F. Rogers, and N. I. Zheludev, Light Sci. Appl. 7, 17157 (2018).

[40] D. Correas-Serrano, A. Alù, and J. S. Gomez-Diaz, Phys. Rev. B 96, 075436 (2017).

[41] J. S. Gomez-Diaz, M. Tymchenko, and A. Alù, Phys. Rev. Lett. 114, 233901 (2015).

[42] R. Messina, A. Noto, B. Guizal, and M. Antezza, Phys. Rev. B 95, 125404 (2017).

[43] J. D. Shen, X. L. Liu, and Y. M. Xuan, Phys. Rev. Appl. 10, 034029 (2018).

[44] Y. Zhang, H. L. Yi, and H. P. Tan, ACS Photon. 5, 3739 (2018).

[45] J. S. Gomez-Diaz, M. Tymchenko, and A. Alù, Opt. Mater. Express 5, 2313 (2015). 
[46] J. Christensen, A. Manjavacas, S. Thongrattanasiri, F. H. L. Koppens, and F. J. García de Abajo, ACS Nano 6, 431 (2011).

[47] S. Albaladejo, R. Gómez-Medina, L. S. Froufe-Pérez, H. Marinchio, R. Carminati, J. F. Torrado, G. Armelles, A. GarcíaMartín, and J. J. Sáenz, Opt. Express 18, 3556 (2010).

[48] L. Novotny and B. Hecht, Principles of Nano-optics (Cambridge University Press, Cambridge, 2012).

[49] A. Lakhatakia, Int. J. Infrared Milli. 13, 161 (1992).

[50] G. W. Hanson, IEEE T. Antenn. Propag. 56, 747 (2008).

[51] L. X. Ge, Y. P. Cang, K. Gong, L. H. Zhou, D. Q. Yu, and Y. S. Luo, AIPAdv. 8, 085321 (2018).

[52] L. A. Falkovsky, J. Phys. Conf. Ser. 129, 012004 (2008).

[53] Handbook of Optical Constants of Solids, edited by E. Palik (Academic, New York, 1998).

[54] J. S. Gomez-Diaz and A. Alù, ACS Photon. 3, 2211 (2016).
[55] A. Narayanaswamy, S. Shen, and G. Chen, Phys. Rev. B 78, 115303 (2008).

[56] E. Rousseau, A. Siria, G. Jourdan, S. Volz, F. Comin, J. Chevrier, and J.-J. Greffet, Nat. Photon. 3, 514 (2009).

[57] S. Shen, A. Mavrokefalos, P. Sambegoro, and G. Chen, Appl. Phys. Lett. 100, 233114 (2012).

[58] P. J. van Zwol, L. Ranno, and J. Chevrier, Phys. Rev. Lett. 108, 234301 (2012).

[59] P. J. van Zwol, S. Thiele, C. Berger, W. A. de Heer, and J. Chevrier, Phys. Rev. Lett. 109, 264301 (2012).

[60] M. G. Silveirinha, S. A. H. Gangaraj, G. W. Hanson, and M. Antezza, Phys. Rev. A 97, 022509 (2018).

[61] S. Pakniyat, A. M. Holmes, G. W. Hanson, S. A. H. Gangaraj, M. Antezza, M. G. Silveirinha, S. Jam, and F. Monticone, arXiv:1902.09002v1.

[62] A. Ott, R. Messina, P. Ben-Abdallah, and S.-A. Biehs, Appl. Phys. Lett. 114, 163105 (2019). 